\title{
Prevalence of Hypertension among Obese Children of Private Schools - A Cross-Sectional Study
}

\author{
Anna Tanya Valiyaparambil ${ }^{1}$, Sneha Katke ${ }^{2}$, Manal Anthikat ${ }^{3}$, \\ Ronald Prabhakar ${ }^{4}$, Sachin Maghade ${ }^{5}$ \\ ${ }^{1}$ Intern, ${ }^{2,4}$ Professor, ${ }^{3,5}$ Assistant Professor, \\ MMC. College of Physiotherapy, Wanless Hospital, Miraj, Maharashtra, India
}

Corresponding Author: Anna Tanya Valiyaparambil

\begin{abstract}
Background: Hypertension in childhood, are typically uncommon, as most of the children are asymptomatic. Keeping a check on blood pressure in children can help to identify underlying heath condition. Childhood obesity is associated with its high risk of, cardiovascular diseases, premature death, and disability in adulthood. It is also associated with its future risk of breathing difficulties, fractures, HTN, insulin resistance and psychological effects. This study was done to document the prevalence of hypertension among private school children aged 5-10 years in the state of Maharashtra, India

Objective: To evaluate prevalence of hypertension with obese children of private schools and to find the correlation of hypertension and obesity in children of private schools.

Methods: This cross-sectional study was undertaken on 600 children aged 5 to 10 years of both sexes from private schools in Sangli district, Maharashtra. Prevalence of hypertension was estimated according to cut-off for Indian children. The prevalence of hypertension was compared for different age groups and gender

Results: Data was collected from 157 children consisting of 62 females and 95 males. Statistical analysis was done using Pearson's correlation coefficient test

Conclusion: The prevalence of hypertension was $28 \%$ with $(\mathrm{p}<0.001), 19.7 \%$ were in prehypertensive and $52.2 \%$ were normal. The prevalence was higher in females than in males. The study showed a positive correlation with $(\mathrm{r}=0.61)$. The prevalence of hypertension increased with BMI and age in both sexes.
\end{abstract}

Keywords: Hypertension, obesity, school children, private schools.

\section{INTRODUCTION}

Hypertension is a severe, noninfectious disease and an important risk factor in the causation of CHD, stroke, renal disease and retinal injuries and other cardiovascular complications. ${ }^{[1-3]}$ The disease is a silent threat to the health of individuals all over the world ${ }^{[4]}$. It is the typical vascular disorder, contributing to a significant public health challenge to population in socio-economic transition ${ }^{[1]}$. Pediatric HTN is an upcoming issue in the developed as well as developing countries with prevalence of $\sim 3.5 \%$ in children ${ }^{[2,4,5]}$.

$1 / 3^{\text {rd }}$ of school going children to be overweight/obese, with the proportion significantly higher in private schools compared to government schools. Socioeconomic status and lifestyle factors like reduced physical activity, escalated intake of junk foods and travelling to schools by buses may be the major factors of overweight/obesity among private school children. BMI was significantly higher in private school children than in government 
school children ${ }^{[6]}$. Prevalence of obesity in children ranges up to $2.9 \%-14.3 \%$ according to recent studies done in $2017^{[7]}$. The important risk factor of HTN is obesity and family history ${ }^{[3-4]}$. Therefore, regular BP measurement is extremely necessary in children for early detection, intervention, and prevention of complications due to hypertension ${ }^{[4]}$. The onset of puberty is related with increases in systolic and diastolic blood pressures ${ }^{[8]}$.

A study was undertaken to know the prevalence of hypertension among urban school children aged 5-10 years in North India in Faridabad, Haryana. A total of 5636 children aged 5 to 10 years from both sexes undergone blood pressure, height and weight measurement and the prevalence of hypertension and pre-hypertension was compared for different body mass index categories and gender. Overall, the prevalence of hypertension was $19.7 \%$, while $13.4 \%$ children were in prehypertensive range ${ }^{[9]}$. Another study was done in southern India to determine normal blood pressure in apparently healthy, asymptomatic school children in the age group of 6-16years. They concluded that the blood pressure tends to increase with age, weight, height, and BMI. The blood pressure values (SBP and DBP) increases grossly after 11years of age. The students with positive family history of hypertension had higher valve when compared to another student. The BP of children and adolescents can be assessed using the reference table according to age ${ }^{[2]}$.

Aim of this study is to find out the prevalence of hypertension among obese children aged 5-10 years of private schools. There are two objectives of this study, which are to evaluate prevalence of hypertension and to find the correlation of hypertension and obesity among children of private schools in Maharashtra.

\section{MATERIALS AND METHODS}

This cross-sectional observational study was conducted in 157 obese school children aged 5-10 years of Private schools in Sangli district, Maharashtra. Ethical clearance was obtained from the institutional ethical committee of MMC. College of Physiotherapy, Wanless Hospital, Miraj, Maharashtra. Permission from the concern school principals was taken. Purposive sampling technique was used. A written accent form was taken from all the study participants. The Inclusion criteria for the study were, subjects with BMI $>95^{\text {th }}$ percentile. systemically healthy students, with no history of mental disorder or illness, age group 5 years-10 years. Both boys and girls. Children with Recent cardiac, pulmonary, neurological or musculoskeletal surgeries, systemic illness and co-morbidities were excluded from the study to avoid its influence on obesity.

All the children who were eligible were selected for the measurement of their height and weight. Height was measured with MCP stature meter while the child standing on barefoot with head in Frankfurt plane position.

Weight was measured using OMRON weighing scale with the child wearing only school uniform (without shoes). Three measures for each were obtained and mean of the measure was used for body mass index (BMI) calculation BMI to be calculated using the formula weight $(\mathrm{kg}) /$ height $(\mathrm{m})^{[2] .}$ BMI classification according to Indian academy of Pediatrics As per recommendation of Khadilkar, et al criteria, children were from 5- 17 years ${ }^{[10]}$.

Subjects with $\mathrm{BMI}>95^{\text {th }}$ percentile was selected for further study. Diamond mercurial deluxe BP monitor with MSI Microtone stethoscope was used for measuring blood pressure with appropriate cuff for the arm length. Three readings were measured and mean was considered as final reading. Hypertension classification according to Indian academy of Pediatrics [8]. Children were classified as per recommendation of Raj $M$ et al blood pressure distribution for Indian children ${ }^{[8]}$. 


\section{Statistical analysis:}

Data was collected from 157 children. Statistical analysis was done by SPSS software version 20.0 using Pearson's correlation coefficient test. $\mathrm{P}<0.001$ was considered to be statistically significant.

\section{RESULT}

The study included 157 school children from which 62 were females and 95 were males.

Table 1 shows Age wise prevalence of hypertensive children of private schools. It was found that in 5 years, children, prevalence of hypertension was $20 \%$. In 6 years, children, prevalence of hypertension was $25.5 \%$. In 7 years, children, prevalence of hypertension was $19.4 \%$. In 8 years, children, prevalence of hypertension was $42.1 \%$. In 9 years, children, prevalence of hypertension was $35 \%$. In 10 years, children, prevalence of hypertension was $35.3 \%$. Table 2 depicts gender wise prevalence of hypertensive children of private schools. It was found that female children prevalence of hypertension was $32.3 \%$ and in male children, prevalence of hypertension was $25.3 \%$. Table 3 depicts Correlation between HTN and obesity in children of private schools which Correlation analyses revealed a strong positive relationship between HTN and obesity in children of private schools $(\mathrm{r}=0.61, \mathrm{p}<0.001)$. So, it indicates that as obesity increases, HTN in children of private schools increases

Graph 1 shows average BMI of children of private schools according to their age showing 5 years was $20.48 \mathrm{~kg} / \mathrm{m}^{2 \mathrm{c}}$, 6 years was $21.1 \mathrm{~kg} / \mathrm{m}^{2}, 7$ years was 21.68 $\mathrm{kg} / \mathrm{m}^{2}, 8$ years was $21.72 \mathrm{~kg} / \mathrm{m}^{2}, 9$ years was $23.71 \mathrm{~kg} / \mathrm{m}^{2}$ and that of 10 years was 22.77 $\mathrm{kg} / \mathrm{m}^{2}$. Graph 2 represents distribution of HTN of children of private schools, that, there were $82(52.2 \%)$ children of private school having normal BP, 31(19.7\%) children were prehypertensive while $44(28 \%)$ children were hypertensive.

Table 1: Age wise prevalence of hypertensive children of private schools
\begin{tabular}{|l|l|l|l|l|l|l|l|l|}
\hline \multirow{2}{*}{ Age } & Normal & Prehypertensive & \multicolumn{1}{|l|}{ Hypertensive } & \multicolumn{2}{|c|}{ Total } \\
\cline { 2 - 10 } & Frequency & $\%$ & Frequency & $\%$ & Frequency & $\%$ & Frequency & $\%$ \\
\hline 5 years & 5 & 50.0 & 3 & 30.0 & 2 & 20.0 & 10 & 6.4 \\
\hline 6 years & 30 & 54.5 & 11 & 20.0 & 14 & 25.5 & 55 & 35.0 \\
\hline 7 years & 24 & 66.7 & 5 & 13.9 & 7 & 19.4 & 36 & 22.9 \\
\hline 8 years & 8 & 42.1 & 3 & 15.8 & 8 & 42.1 & 19 & 12.1 \\
\hline 9 years & 8 & 40.0 & 5 & 25.0 & 7 & 35.0 & 20 & 12.7 \\
\hline 10 years & 7 & 41.2 & 4 & 23.5 & 6 & 35.3 & 17 & 10.8 \\
\hline Total & 82 & 52.2 & 31 & 19.7 & 44 & 28.0 & 157 & 100.0 \\
\hline
\end{tabular}

Table 2: Gender wise prevalence of hypertensive children of private schools

\begin{tabular}{|l|l|l|l|l|l|l|l|l|}
\hline \multirow{2}{*}{ Gender } & Normal & \multicolumn{2}{|c|}{ Prehypertensive } & \multicolumn{2}{|l|}{ Hypertensive } & \multicolumn{1}{|c|}{ Total } \\
\cline { 2 - 10 } & Frequency & $\mathbf{\%}$ & Frequency & $\mathbf{\%}$ & Frequency & \% & Frequency & \% \\
\hline Females & 30 & 48.4 & 12 & 19.4 & 20 & 32.3 & 62 & 39.5 \\
\hline Males & 52 & 54.7 & 19 & 20.0 & 24 & 25.3 & 95 & 60.5 \\
\hline Total & 82 & 52.2 & 31 & 19.7 & 44 & 28.0 & 157 & 100.0 \\
\hline
\end{tabular}

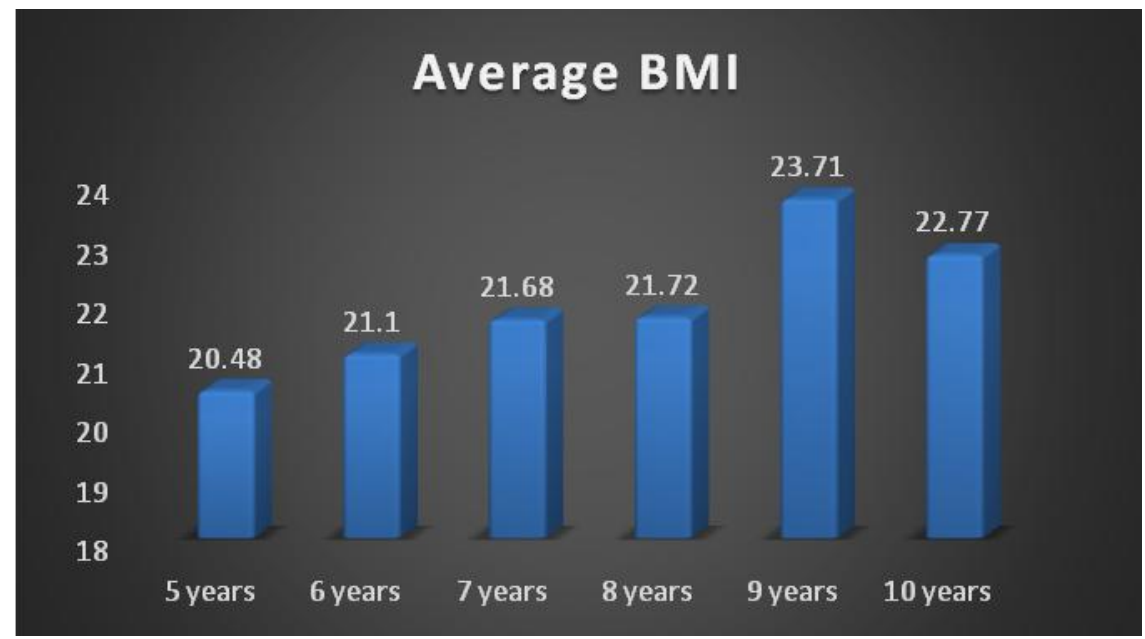

Graph 1: Bar diagram representing average BMI of children of private schools 




Graph 2: Bar diagram representing distribution of HTN of children of private schools

Table 3: Correlation between HTN and obesity in children of private schools

\begin{tabular}{|l|l|}
\hline BMI & BP percentile \\
\hline Pearson Correlation & $\mathrm{p}$ value \\
\hline 0.61 & $<0.001$ \\
\hline
\end{tabular}

\section{DISCUSSION}

Six hundred subjects returned with positive parental consent and were screened for blood pressure. 157 children were found obese. The present study highlights the prevalence of hypertension among 157 (26.2\%) obese school children aged 5-10 years from Sangli district Maharashtra. As there was strict lockdown restriction across state and districts amid Covid-19 pandemic, obese children were called in the batch of 3 in respective schools to avoid mass gathering for BP measurement. All norms of social distancing and sanitization of BP cuffs after each child were followed strictly.

About $(28 \%)$ of the children were detected to be hypertensive, $(19.7 \%)$ were in pre-hypertensive and (52.2\%) were in normal category. From which hypertensive $(32.3 \%)$ and $(25.3 \%)$ females and males respectively and prehypertensive $(19.4 \%)$ and $(20.0 \%)$ females and males respectively. More females were documented to be hypertensive than males of same age where as more males were prehypertensive than females of same age. In 5 years, children, prevalence of hypertension and prehypertension was $20 \%$ and $30 \%$ respectively. In 6 years, children, prevalence of hypertension and prehypertension was $25.5 \%$ and $20 \%$ respectively. In 7 years, children, prevalence of hypertension and prehypertension was $19.4 \%$ and $13.9 \%$ respectively. In 8 years, children, prevalence of hypertension and prehypertension was $42.1 \%$ and $15.8 \%$ respectively. In 9 years, children, prevalence of hypertension and prehypertension was $35 \%$ and 25.0 respectively. In 10 years, children, prevalence of hypertension and prehypertension was $35.3 \%$ and 23.5 respectively. There was increase in prevalence of hypertension and prehypertension with age and BMI status. Correlation analyses revealed a strong positive relationship between HTN and obesity in children of private schools $(\mathrm{r}=0.61, \mathrm{p}<0.001)$. So, it indicates that as obesity increases, HTN in children of private schools increases.

Similarly, results were seen in studies done among school going children by Behram shahid et al. ${ }^{[11]}$, Manoja Kumar Das et al ${ }^{[9]}$. and N.K. Anand et $a l^{[4]}$ and reported hypertension was $7.5 \%, 19.7 \%$ and $3.5 \%$ of the study population, respectively. Similar observations were reported by study from Haryana, India, were the prevalence of hypertension to be $19.1 \%$ in boys and $20.3 \%$ in girls ${ }^{[9]}$. Among 6-14 years old children in Amritsar, hypertension was documented in $7.5 \%$ of health children (boys $=8.33 \%$ and girls $=6.52 \%$ ). The prevalence of hypertension increased with age in boys till10 years of age and in girls till 11 years of age and then dropped till 14 
years of age ${ }^{[12]}$. In Bhopal, among the children aged 14-17 years, a highly significant association $(P<0.01)$ of sex, BMI status of the population and sedentary lifestyle as socioeconomic status and lifestyle factors like reduced physical activity, escalated intake of junk foods and travelling to schools by buses may be the major factors of overweight/obesity ${ }^{[6]}$.

This study indicates the high prevalence $(p<0.001)$ of hypertension in children of 5-10 years in Sangli district, India. With increase in age and BMI, the prevalence of hypertension increased in both sexes. As this study was conducted in 2021, the schools, playground, parks etc., were closed due COVID-19 pandemic there is a decreased physical activity and increased sedentary life style of the child, might be a reason of increased prevalence of obesity. The findings suggest for systematic nationwide documentation of the prevalence of hypertension and its relationship with the risk factors to identify suitable preventive measures. Also, it mandates blood pressure check-up for children during routine Pediatrics clinical practice and school health check-up, including dietary advice, exercise protocol according to their age and heart rate should be prescribed.

Meyer AA et al ${ }^{[13]}$, studies show there was an Improvement of early vascular changes and cardiovascular risk factors in obese children after a six-month exercise program. Exercises were conducted 3 times/week: on Mondays, swimming and aqua aerobic training (60 $\mathrm{min})$; Wednesdays, sports games (90 min); and Fridays, walking (60 min), by qualified coaches and physiotherapists. Intervention produced a significant decrease in systolic mean BP and an increase in physical fitness data (maximum work capacity [Wmax] ${ }^{[13]}$.

There are few limitations in this study. This study was conducted with the available students of private schools due to COVID-19 pandemic restrictions and guidelines; all schools were closed due to states were having strict lockdown rules and regulations in India. Only private schools of
Sangli district were included and subjects from rural schools were not. Experimental Studies on hypertensive and prehypertensive obese children can be done by giving detailed exercise protocol to reduce BMI of obese school children of private schools and recording their blood pressure readings before intervention and after intervention once their BMI is between $3^{\text {rd }}-85^{\text {th }}$ percentile according to their age group. Similar study can be done on lower and middle socioeconomic class children.

\section{CONCLUSION}

In this study, there is a prevalence of hypertension in obese children of private schools among 5-10 years of age is $28 \%$. There is a high significant association $\mathrm{p}<0.001$ of BMI and blood pressure was seen. There is a significantly positive correlation analyses revealed a strong positive relationship between hypertension and obesity in children of private schools $(\mathrm{r}=0.61)$, which indicates that as obesity increases, hypertension in children increases. It is necessary to keep a check on blood pressure for children during routine Pediatrics clinical follow up, school health check-up, and detailed exercise protocol to be advised by a qualified physiotherapist like swimming, sports, outdoor games, walking etc., to maintain normal body mass and BMI which will help to prevent future cardiovascular complications.

\section{ACKNOWLEDGEMENT}

It's my great pleasure and privilege to express my deep-felt gratitude to our respected Principal sir Dr. Ronald Prabhakar and Guide Dr. Sneha Katke who immensely helped me and rendered their advice, precious time, constant encouragement, knowledge and relevant information regarding my study, and whose suggestion and guidance has enlightened me on this subject. I am also great full to Dr. Amit Nagrale Professor dept. Of community medicine, Miraj Medical Centre, College of physiotherapy, Wanless Hospital, for help in the data analysis of this study. I express my 
Anna Tanya Valiyaparambil et.al. Prevalence of hypertension among obese children of private schools - a cross-sectional study.

sincere thanks to all the teaching \& nonteaching staff of the Miraj Medical Centre, College of Physiotherapy.

\section{Conflict of Interest: None}

\section{Source of Funding: None}

\section{Ethical Approval: Approved}

\section{REFERENCES}

1. K park. Park's textbook of preventive and social medicine. $24^{\text {th }}$ ed. Banarsidas Bhanot Publishers. Madhya Pradesh; 2017. p. 391

2. Sayeemuddin M, Sharma D, Pandita A, Sultana T, Shastri S. Blood pressure profile in school children (6-16 years) of Southern India: a prospective observational study. Frontiers in pediatrics. 2015 Mar 31;3:24.

3. Uwaezuoke SN, Okoli CV, Ubesie AC, Ikefuna AN. Primary HTN among a population of Nigerian secondary school adolescents: Prevalence and correlation with anthropometric indices: A cross-sectional study. Nigerian journal of clinical practice. 2016;19(5):649-54.

4. Anand NK, Tandon L. Prevalence of HTN in school going children. Indian pediatrics. 1996 May; 33:377-81.

5. Flynn JT, Kaelber DC, Baker-Smith CM, Blowey D, Carroll AE, Daniels SR, de Ferranti SD, Dionne JM, Falkner B, Flinn SK, Gidding SS. Clinical practice guideline for screening and management of high blood pressure in children and adolescents. Pediatrics. 2017 Sep 1;140(3): e20171904.

6. Patnaik L, Pattanaik S, Sahu T, Rao EV. Overweight and obesity among adolescentsa comparative study between government and private schools. Indian pediatrics. 2015 Sep 1;52(9):779-81.

7. Eshwar TM, Chudasama RK, Eshwar ST, Thakrar D. Prevalence of obesity and overweight and their comparison by three growth standards among affluent school students aged 8-18 years in Rajkot. Indian journal of public health. 2017 Jan 1;61(1):51

8. Raj M, Sundaram KR, Paul M, Kumar RK. Blood pressure distribution in Indian children. Indian pediatrics. 2010 Jun 1;47(6):477-85.

9. Das MK, Bhatia V, Sibal A. Prevalence of HTN in urban school children aged 5 to 10 years in North India. International Journal of Contemporary Pediatrics. 2017 Nov;4(6):2055

10. Khadilkar VV, Khadilkar AV. Revised Indian Academy of Pediatrics 2015 growth charts for height, weight and body mass index for 5-18-year-old Indian children. Indian journal of endocrinology and metabolism. $2015 \mathrm{Jul} ; 19(4): 470$.

11. Shahid B, Jalal MA, Waseem M, Shahid H, Mehboob-Ur-Rehman M. Prevalence of obesity in school going adolescents and its association with HTN. Pakistan journal of medical \& health sciences. 2017 Jul 1;11(3):1082-4.

12. Kaur A, Kaur P, Kaur N, Kumari K, Sidhu S. Variation in blood pressure among school children of Amritsar (Punjab). The Anthropologist. 2005 Jul 1;7(3):201-4.

13. Meyer AA, Kundt G, Lenschow U, SchuffWerner P, Kienast W. Improvement of early vascular changes and cardiovascular risk factors in obese children after a six-month exercise program. Journal of the American College of Cardiology. 2006 Nov 7;48(9):1865-70

How to cite this article: Valiyaparambil AT, Katke S, Anthikat $M$ et.al. Prevalence of hypertension among obese children of private schools - a cross-sectional study. Int $J$ Health Sci Res. 2021; 11(7): 31-36. DOI: https://doi. Org/10.52403/ijhsr.20210705 